\title{
NDIR Gas Measurement in Harsh Environments by Advanced IR Components
}

\author{
Steffen Biermann ${ }^{1}$, André Magi $^{1}$, Falk Liebold ${ }^{1}$, Patrick Sachse ${ }^{1}$ \\ ${ }^{1}$ Micro-Hybrid Electronic GmbH, Heinrich-Hertz-Str. 8, 07629 Hermsdorf, Germany, \\ s.biermann@micro-hybrid.de
}

\begin{abstract}
Summary
Based on the well-known optical NDIR gas measurement principle Micro-Hybrid developed an advanced gas sensor which works in harsh environments like extraordinary moistures and high temperatures up to $200{ }^{\circ} \mathrm{C}$. A field of application is controlling the $\mathrm{CO}_{2}$ atmosphere and the measurement of the humidity in sterilisable incubators. Core components of the developed gas sensor are highly effective and high temperature stable infrared emitter and detector MEMS chips. The precondition for long term stability is a real hermetic housing especially under these rough conditions. Common gluing technologies are not suitable for such terms. To achieve these requirements Microhybrid developed sophisticated packaging technologies for the IR-MEMS chips and the housing components which are appropriate for harsh environments stable hermetic sealing like high temperatures, high humilities or solvent atmospheres.
\end{abstract}

Keywords: gas analysis, NDIR gas sensor, hermetic packaging, high temperature, infrared components

\section{Motivation}

NDIR gas sensors are used successfully in a wide range for detecting, measuring and controlling infrared active gases. The applications like environmental engineering, chemical industry, biotechnology and health care are wide diversified regarding the gases and the concentration levels to be measured. The advantages of this technology are the high measuring accuracy, the long term and system stability as well as the robustness of these systems based on the simple construction. However most of these are subject to restrictions concerning the environmental stability, in particular the temperature resistance. The maximum storage and operating temperature of common NDIR gas sensors is limited at around $+60^{\circ} \mathrm{C}$ operation temperature and applications, where the sensor is exposed to higher temperatures are highly restricted. This fact was the intention for Micro-Hybrid to develop a NDIR gas sensor with a distinct extended temperature range up to $200{ }^{\circ} \mathrm{C}$ and for harsh environments like high vapour partial pressure up to $800 \mathrm{mbar}$ and solvent atmospheres. The NDIR gas sensor consists of an IR-Source, a multi-channel IRdetector, the absorption path in the gas chamber, and further electronics (Figure 1).

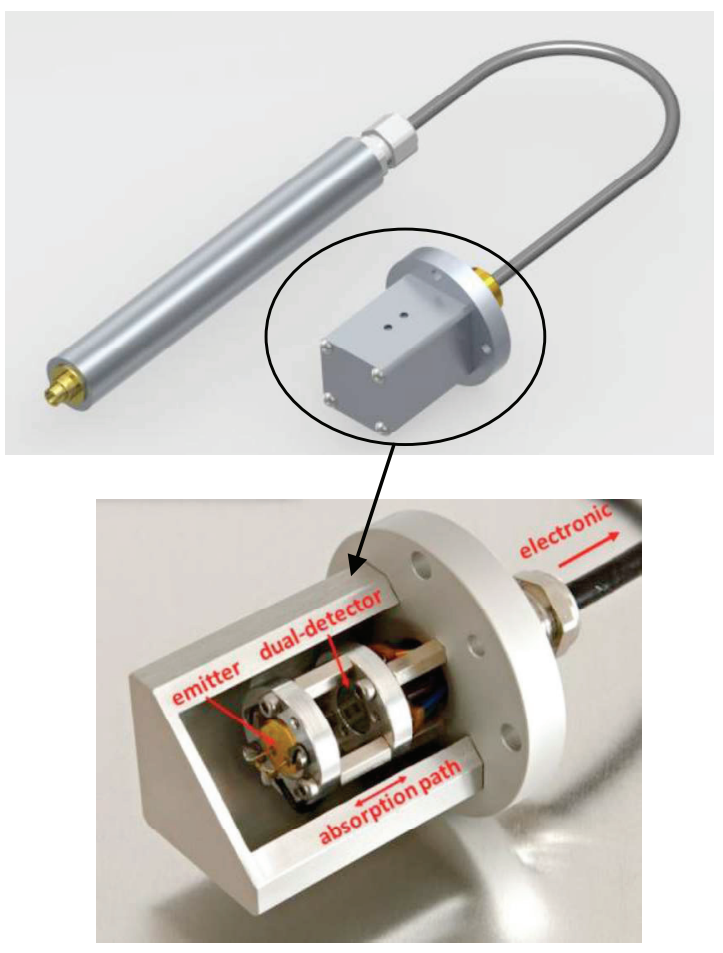

Fig. 1: High temperature $\mathrm{CO}_{2}$ gas sensor module; above CAD model; below cross section of the sensor 


\section{Measurement principle}

The module based on the well-known NDIR (non dispersive infrared) gas measurement principle which is established as a common gas analysis technique. The configuration of the module is designed for quantitative measuring $\mathrm{CO}_{2}$ concentration, especially in high concentrations. Figure 2 shows a principle picture of the module. The absorption path of the measuring chamber is filled with the gas to be investigated. Infrared radiation delivered by a MEMS based IR- source passes through the gas and generates a signal voltage in the thermopile infrared detector.

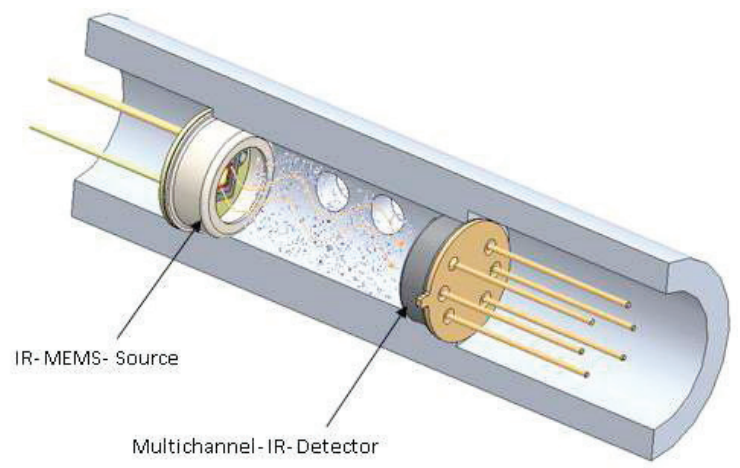

Fig. 2: Principle of the gas measuring chamber

The detector is assembled with narrow band pass filters. The CWL of the filters is fitted to their specific transmission wavelength of the gas which should be detected. The developed gas measurement module contains a special multichannel thermopile detector depending on the measured gases. In figure 3 the absorption of $\mathrm{CO}_{2}$ is shown as a function of the wavelength at room temperature under normal pressure. In the same graph the transmission curve of the used infrared filters is shown.

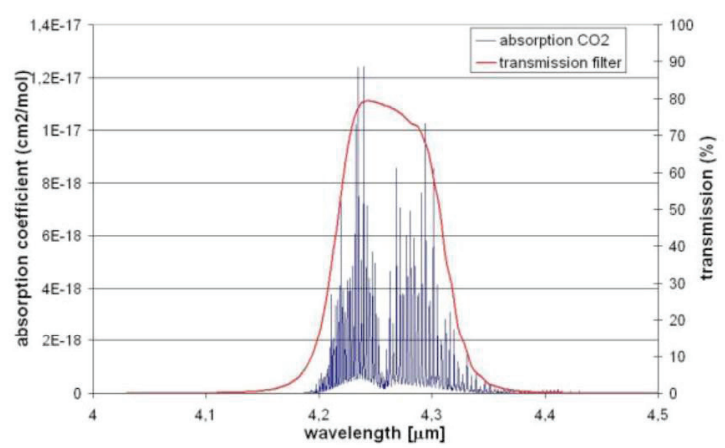

Fig. 3 Absorption of $\mathrm{CO}_{2}$ and transmission curves of the used filter as a function of the wavelength

One of the canals of the detector has a IR-filter which is not coincident with the absorption wavelengths of $\mathrm{CO}_{2}$. It is used as a reference channel to correct possible changes of the intensity of the source that can occur by aging effects or by the influence of dust and smoke. The infrared source is switched on and off periodically. In this way disturbing offset voltages can be eliminated by measuring only the signal differences.

\section{Applications}

The application fields of the new NDIR gas sensor are based on its resistivity against harsh environment conditions. An example is the use of this sensor for the measurement respectively control of the $\mathrm{CO}_{2}$ atmosphere and the humidity in $\mathrm{CO}_{2}$ incubators. For an optimal growth of cell cultures are specific and long term stable environment conditions like temperature, humidity and especially the $\mathrm{pH}$ value necessary. The $\mathrm{pH}$ number will be adjusted by the $\mathrm{CO}_{2}$ concentration within the incubator chamber. Since the breeding cells are mostly extreme sensitive against deviations of the $\mathrm{pH}$-value an exact and high stable $\mathrm{CO}_{2}$ adjustment is absolute necessary. Only by ensure such settings the requirements on optimal and reproducible conditions of growth of cell cultures can be guarantee. The common atmospheric conditions are a temperature of $37^{\circ} \mathrm{C}$, a humidity of $95 \%$ rh and a $\mathrm{CO}_{2}$ concentration of 5 vol.- $\%$. The most extreme humidity and temperature conditions can be adjusted and occur with $60{ }^{\circ} \mathrm{C}$ and $95 \%$ rh [2]. The resulting water vapour partial pressure of 190 mbar in combination with this temperature constitutes highest challenges on the system stability of the gas sensors especially the hermetically packaged infrared components. Common packaging technologies which are often based on glue materials like epoxies are not suitable for such harsh terms. Due to the use of $\mathrm{CO}_{2}$ incubators also in highly critical and sensible application fields like:

- bio tissue engineering,

- in vitro fertilisation (IVF),

- human and veterinary medicine,

- pharmaceutic industry as well as

- biotechnology

there are very high requirements on the probe safety and consequently also on the reliability and long term stability of the gas sensor. But crucial is the unique feature that the gas sensor is high temperature stable up to $200^{\circ} \mathrm{C}$ which is necessary for the hot air sterilization of the incubator according DIN 58947 at minimum $180^{\circ} \mathrm{C}$. This kind of sterilization is the most safety and reliable method to eliminate toxic and hazardous bacteria and spores to decontaminate the chamber of the incubator. The exposer of this temperature level and temperature change represents an 
extraordinary thermomechanical stress for the whole assembly of the gas sensor inclusive the integrated infrared optical components. The system can only withstand these condition based on the specific developed organic free packaging technologies.

Further application fields are also given e. g. in the case of the measurement of concentrations of solvent atmospheres at higher temperatures. According the law of Arrhenius under these conditions are existing accelerated reaction rates. This leads to a significant boosted chemical exposure for the hermetically assembly joins and especially also for the optical infrared filters. Due to the basically unknown resistance of the optical filter deposition against solvent atmospheres and high humidity there are time-consuming tests necessary. To prevent such expensive investigations for each different filter a special construction design for the infrared components was developed.

\section{Sensor requirements}

During the structural sensor design beside of thermomechanical aspects it needs to be also the focus on temperature dependencies and effects of the thermoelectrically elements on optical transfer elements. In the following some selected effects and their impacts will be described.

\section{Thermopile element}

The signal voltage $U_{S}$ of the thermopile detector depends strong on the temperature of the element itself. This based on the temperature dependence of the thermoelectrically coefficient [6] and on the effective radiation flux $\Delta \Phi_{\mathrm{S}}$, which is converted into heat on the membrane [3].

$$
U_{S}=R_{V} \cdot \Delta \Phi_{S} \sim R_{V}\left(\Phi_{\text {in }}-\sigma \cdot T_{\text {Det }}^{4}\right)
$$

Equation 1 describes this correlation with the following parameters schematically.

- $\Delta \Phi_{\mathrm{S}} \quad$ effective radiation flux

- $\Phi_{\text {in }}$ whole incoming radiation flux

- $\sigma$ Stefan-Bolzmann constant

- $R_{V} \quad$ voltage sensitivity

- $\mathrm{T}_{\text {Det }}$ absolute detector temperature

Most of these parameters are basically depending on the temperature and the wavelength.



Fig. 4: Normalized thermopile signal as a function of the ambient temperature

In Figure 4 the detector output signal is shown as a function of the ambient temperature. As obvious to see over a temperature range from $30{ }^{\circ} \mathrm{C}$ to $190{ }^{\circ} \mathrm{C}$ the signal drops over $40 \%$. This effect needs to be considered during the gas sensor design. In our case the thermopile detector consists of highly effective $\mathrm{BiSb} / \mathrm{Sb}$ thermocouples which enable a high sensitivity and a sufficient signal to noise ratio at high temperatures, too.

A further disturbing effect of thermoelectrically based measurement systems is the so called thermos-shock effect. It describes the signal behaviour after rapid thermal changes of the ambient respectively the housing of the detector. In such cases the detector system which consists of a socket, thermopile MEMS chips, a cap and optical interference filter becomes out of equilibrium. As a result of this thermal imbalance high deficient signal changes occur which can exceed the actual measurement effect many times. During the subsequent thermal compensation process it is not possible to analyse the true signal due to the heavy signal changes. The characteristic and the value of this effect depend on the detector construction and the thermal assembly situation. Minor thermal differences lead to highly different thermal-shock behaviours of the detector. By using an internal aperture and selected thermal conductive glues for the inner detector assembly of the infrared chips and the optical infrared filters as well as the well-considered assembly of the detector in the gas measurement cell the effect is controlled in the current gas system construction. Figure 5 shows the signal response of three different detectors after a rapid change of the ambient temperature from $25^{\circ} \mathrm{C}$ to $50^{\circ} \mathrm{C}$. 




Fig. 5: signal response of 3 detectors after a rapid change of the ambient temperature $\Delta T \approx 30 \mathrm{~K}$.

\section{Interference optical filter}

To achieve a wavelength selective sensitivity of the thermopile detector optical narrow bandpass interference filter are arranged in the front of the broadband absorbance thermopile sensor element. The central wavelength $\left(\lambda_{\text {max. }}\right)$ and the full width at half maximum (FWHM) of the filters are fine tuned to the specific measuring task. Conditional on the thermal dilatation of dialectical thin-film layers of the optical filters the spectral transmission properties changes as a function of the ambient temperature [7].

$$
\lambda(T)=\lambda_{0}\left(1+\Delta T \cdot 0,0102 \frac{\%}{K}\right)
$$

The shift of the central wavelength can obtain approximately by equation 2 [10]. If there is a need of exact values a specific spectral measurement over the target temperature range is necessary. Figure 6 shows temperature profiles of different measured infrared filters.

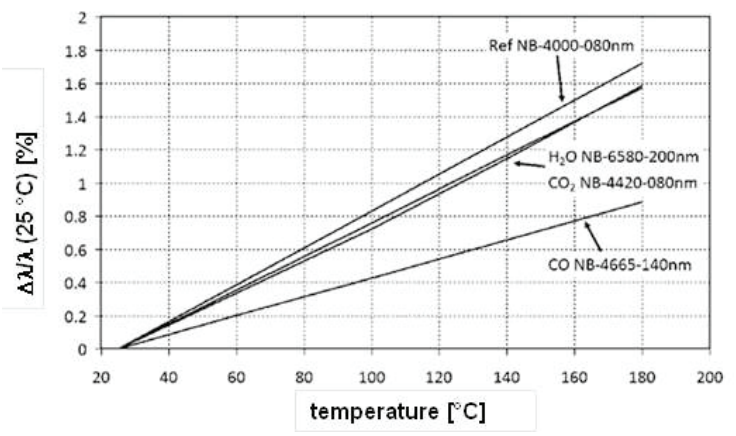

Fig. 6 Percentage change of the central wavelength $\lambda$, as a function of the temperature $T$ for interference filters $4.665 \mu \mathrm{m}, 4.420 \mu \mathrm{m}, 6.580 \mu \mathrm{m}$ and $4.000 \mu \mathrm{m}$ (NB=Narrow-Bandpass).
At the detector design and the selection of the specific interference filter is always a compromise between a high selectivity (=narrow-bandpass filter) and a decent signal resolution of the thermal detector (=broadbandpass filter) necessary. In this context it must be point out that high signal selectivity can also generate negative effects in terms of linearization function if a wide operation temperature range is needed. Also in the case if several gases with different spectral absorption coefficients are simultaneously to be measured by a multichannel detector a compromise between the optimal absorption lengths of the measurement cell, the selected absorption wavelength of the measurement gases and the nonlinear absorption behaviour of these gases as a function of their concentration must be considered.

For example:

In a special application it is necessary to measure high $\mathrm{CO}_{2}$ concentration in combination with other Gases over a wide temperature range. For another gas a path length of $50 \mathrm{~mm}$ is required. To measure $\mathrm{CO}_{2}$, an infrared filter with a CWL on $4.42 \mu \mathrm{m}$ is needed. In this case the linearization function shows a high temperature dependency due to the central wavelength shift of the infrared filter (Fig. 7). This fact requires an elaborate calibration process.

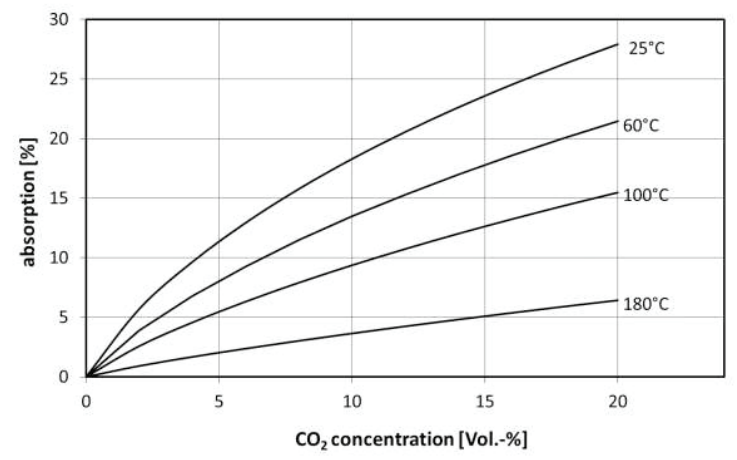

Fig. 7: Linearization function depending on temperature of an setup with $50 \mathrm{~mm}$ path length an $\mathrm{CO}_{2}$ interference filter with CWL $4.42 \mu \mathrm{m}$

Furthermore, for applications with wide operation temperature range it is technically possible to optimize the temperature CWL shift to a minimum by a special filter design and layer processing at the filter manufacturer. 


\section{Infrared sources}

For infrared radiation sources thermal emitters are generally used. According to the application different types of sources with different assemblies are be deployed. In contrast to incandescent lamps Micro-Hybrid's thin film IR sources can operate long time stable on air with membrane temperatures up to $850^{\circ} \mathrm{C}$. The thin film sources (MEMS based) have a similar structure like thermopiles, whereby the resistive heater element is spattered as a thin film layer on the membrane. The heater element can be structured in different ways like e.g. platinum meander or with a nano-amorphous diamondlike carbon (NAC) layer.

The used Micro-Hybrid emitter based on thermally stable nano-amorphous diamondlike carbon coatings. This structure enables high membrane temperatures up to $850{ }^{\circ} \mathrm{C}$ as well as high radiation intensities. Ongoing developments show further enhancements of the emitter. The emission surface is based on advanced silicon nanostructures. Therefore a spectral broadband emission coefficient near 1 is achievable as an ideal blackbody (Fig. 8 and Fig. 9).

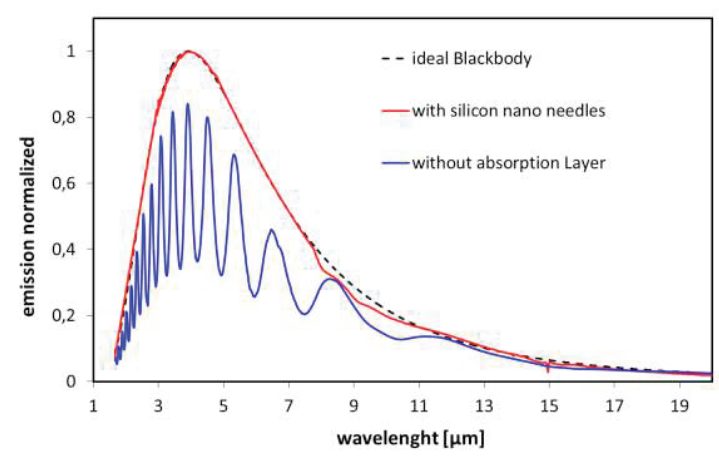

Fig. 8: Emission spectrum of MEMS emitter with and without an emission layer consisting of silicon nano-needles

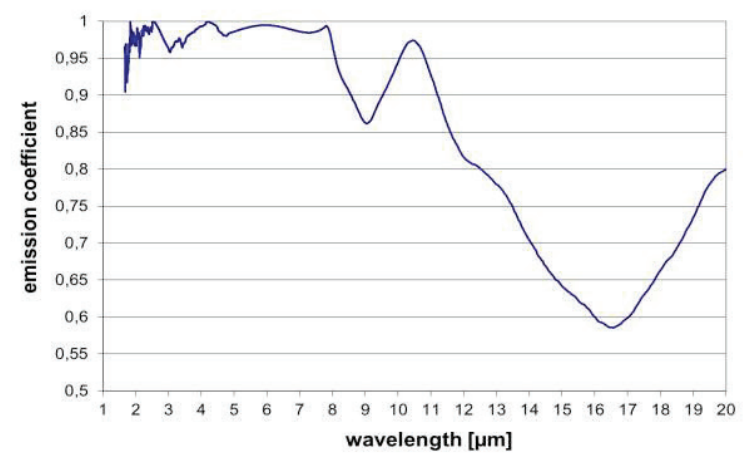

Fig. 9: Emission coefficient of MEMS emitter with an emission layer consisting of silicon nano-needles
A possibility to increase the emission coefficient is shown in figure 10. In this example the surface is heavily increased by the silicon nano-needles (black silicon) and the emission characteristic is optimized by thin film coating with platinum as well a final coating with $\mathrm{SiO}_{2}$ (Fig. 11). Due to the low thermal mass of the black Si emission layer there is up to $10 \mathrm{~Hz}$ practically no influence on the time response of the system.

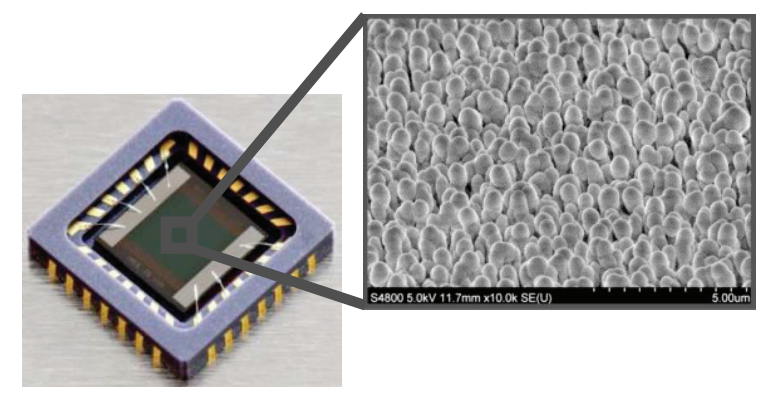

Fig. 10: MEMS emitter with an additionally emission layer of silicon nanoneedles deposited with $P$ t and a passivation with $\mathrm{SiO}_{2}$ [5]

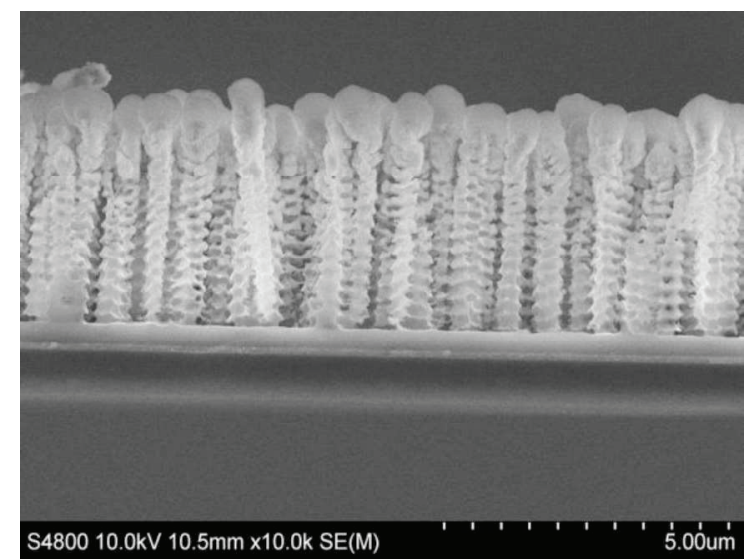

Fig. 11: Cross section of the black silicon emission layer of MEMS emitter

Only a small part of the electrical input power is converted into infrared radiation. A large percentage of the electrical input power is dissipated by thermal conduction into the socket and through the gas atmosphere. By using a thermal isolation for the emitter chip the thermal conductivity can be counteracted. In a sealed system the thermal loss can be decreased by backfilling the sources with thermal isolated gases like Krypton. Such a thermal isolated backfill gas has also an influence of the time constant, which will be increased (Fig 12). 


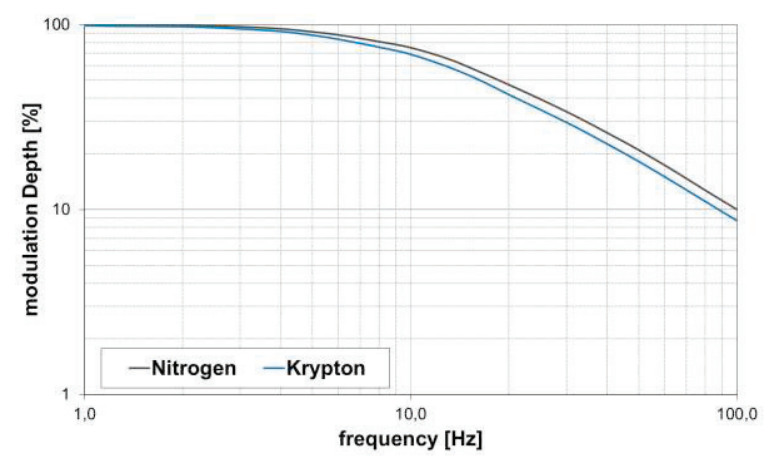

Fig. 12: Frequency response characteristic of standard NAC emitter with different backfill gases

In total less electrical input power has to be used to heat up the membrane to the same temperature level. Hereby highly effective infrared emitter can be assembled, which has a high specific radiation, good modulation behavior and a long lifetime.

\section{Packaging of infrared components}

To guarantee a long term stability of the infrared components even used in harsh environments a real hermetic packaging technology is necessary. Micro-Hybrid developed such technologies based on welding and soldering process steps. Contrary to adhesion joints there is no permeation into the case of the components existing which leads to drift behaviour and in the case of oxygen at high temperatures to a breakdown of the infrared MEMS chips. Especially under high temperature and high humidity conditions gluing materials are not suitable in terms of real hermetically joints. They decline quite fast and lose their quasi hermetic properties. In the context of the soldering technology for the optical coated infrared filters and windows a new metallization and separation technology for free shaped geometries was developed [4]. The intention was to assemble the functional filters inside of the components and protected the inner area and assembly by an outside hermetically soldered infrared window which contains outside of a special thin-film cover and harsh environment resistant optical layer. Both key components detector and emitter contain such a window on top. Figure 13 shows such a typical dual detector for high temperature gas sensor applications with a measurement and a reference channel packaged in a real hermetic TO39 housing [1].

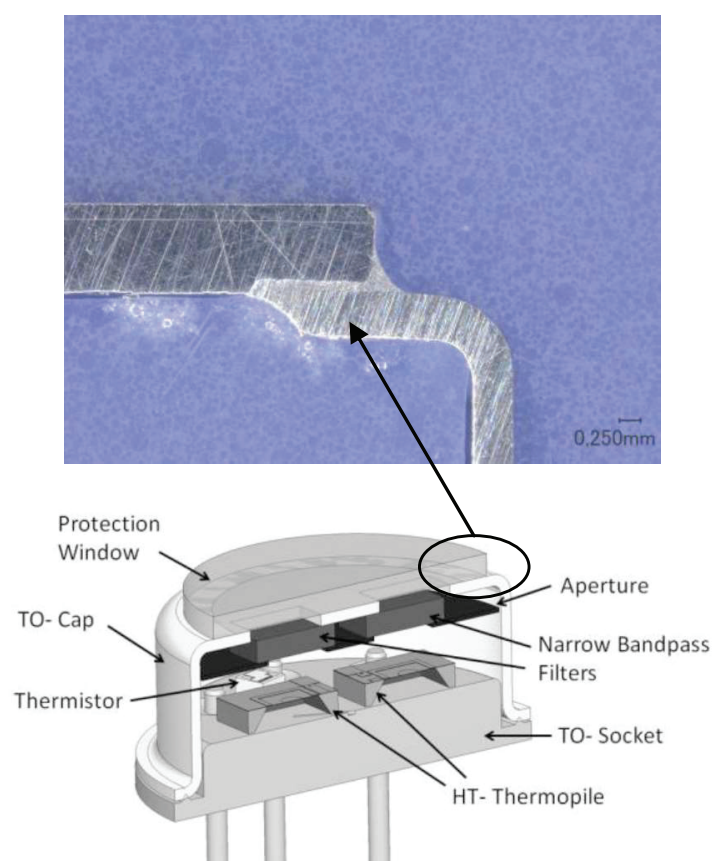

Fig. 13: Dual high temperature thermopile infrared detector with hermetic sealed TO housing by soldered window.

The connection between TO-socket and detector cap is realised by a highly reliable resistance welding process. Backfilling inert gases during this welding process, like dry nitrogen, make these components useful in cold environments down to $-40{ }^{\circ} \mathrm{C}$. Furthermore it is possible to tune the sensor and emitter parameters with different backfill gases. For example the sensitivity of the IRdetector can be increased by backfilling an inert gas with a low thermal conductivity. On the same way the power consumption of the IR- emitters can be decreased. By using gases with a high thermal conductivity the time response of the components can be accelerated. In table 1 typical used backfill gases are summarized.

Tab. 1: Coefficient of thermal conductivity $\lambda$ of various inert gases [9]

\begin{tabular}{|c|c|c|}
\hline Gas & $\boldsymbol{\lambda}[\mathbf{W} / \mathbf{m} / \mathbf{K}]$ & Relation to $\mathbf{N}_{\mathbf{2}}$ \\
\hline $\mathrm{He}$ & 0.143 & $6: 1$ \\
\hline $\mathrm{Ne}$ & 0.046 & $1.9: 1$ \\
\hline $\mathrm{N}_{2}$ & 0.024 & $1: 1$ \\
\hline $\mathrm{Ar}$ & 0.016 & $1: 1.5$ \\
\hline $\mathrm{Kr}$ & 0.0088 & $1: 2.7$ \\
\hline $\mathrm{Xe}$ & 0.0051 & $1: 4.7$ \\
\hline
\end{tabular}

The correlation between the thermal conductivity of the backfill gases and the sensitivity respectively the time constant can used to be evaluating the hermetic behaviour of the components. In this context the high 
thermal conductivity of $\mathrm{He}$ will be used. The procedure is the following:

- Measuring the sensitivity respectively the time constant of the component

- He overpressure storage at 3 bar over e. g. $24 \mathrm{~h}$

- Re-measuring of the component and comparison of the values with the premeasurement

If the component is not $100 \%$ leak tight He will diffuse into the housing and changes the parameters in the direction of lower sensitivity and lower time constant. Due to the long term He overpressure storage this procedure is multiple more sensitive than the standard $\mathrm{He}$ leak-test by using commercial equipment.

\section{Signal conditioning}

As shown in figure 1 the electronic evaluation circuit is separated from the gas measurement cell. The electrical connections are made by a high temperature cable that contains twisted and shielded wires. The distance between electronic and measurement cell can be up to $20 \mathrm{~cm}$ without influencing the signal to noise. The optical part of the sensor is usually located in a constant temperature atmosphere, but the electronic is influenced by room temperature fluctuation of more than $10 \mathrm{~K}$ which has to be considered in the electronic design and signal processing. Usually causes weak emitter power variation a significant change of the $\mathrm{CO}_{2}$ signal. Caused by the nonlinear behaviour between temperature and spectral emissivity the radiation intensity changes differently for measurement and reference channels. The result of this radiation energy change is a zero point error of the gas sensor shown in figure 14.



Fig. 14: Zero point error (black line) and membrane temperature (doted line) as a function of emitter power variation $\pm 5 \%$

\section{System design of the gas sensor}

The dual channel detector and the MEMS emitter are mounted in a hermetic package with a soldered protection window. In each housing of both thermopiles a thermistor is included which is needed to compensate temperature effects. To increase the efficiency both housings are filled with Krypton. Due to this measure the sensitivity of the whole gas sensor increased by $80 \%$ compared with nitrogen for the use as a heat isolator. Therefore it is possible to achieve at low power consumption of $300 \mathrm{~mW}$ and a emitter membrane temperature of nearly $450{ }^{\circ} \mathrm{C}$. In this case the spectral $\mathrm{CO}_{2-}$ and reference channel are close to Planck's intensity maximum. The signal processing electronics is mounted outside the measurement chamber in a low temperature environment $\left(<80^{\circ} \mathrm{C}\right)$.

(see fig. 1). An optical IR-filter (Type NB-4260$180 \mathrm{~nm}$ ) is used to determine the absorption as a function of the $\mathrm{CO}_{2}$ concentration (Fig. 3). For the desired measurement range up to $20 \mathrm{Vol} .-\% \mathrm{CO}_{2}$ only a short absorption path of $3 \mathrm{~mm}$ is needed. Due to the various temperature dependencies of the used components of the $\mathrm{CO}_{2}$ gas measurement sensor a multi field calibration over several days is necessary. To achieve the desired accuracy, the gas sensor is calibrated at different $\mathrm{CO}_{2}$ concentrations and temperatures. The integrated microcontroller calculates the gas concentration by special algorithm based on the analogue nonlinear thermopile signals and the temperature of the measurement cell.

\section{Evaluation of the gas sensor}

The following charts in Figure 15 represent the typical signal behaviour at different temperatures. The signal to noise ratio (SNR) degreases minimally from $42 \mathrm{~dB}$ at $30^{\circ} \mathrm{C}$ to $38 \mathrm{~dB}$ at $180^{\circ} \mathrm{C}$, only. Therefore the sensor is suitable for a wide range of applications.
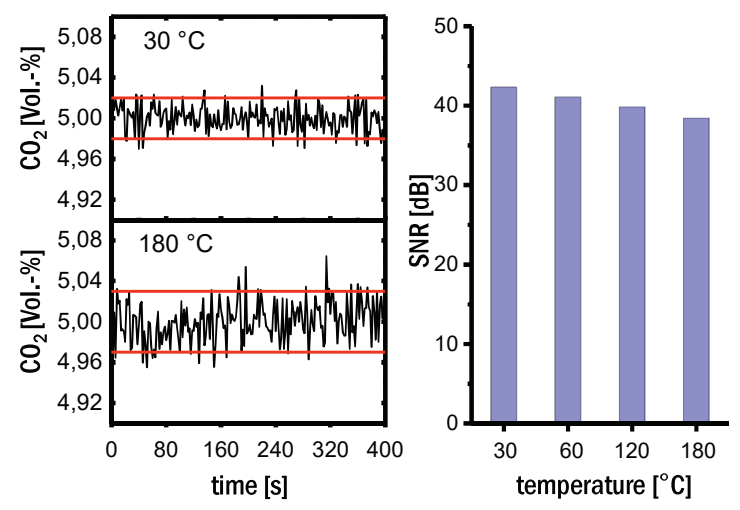

Fig. 15: Typical signal behaviour at 5 Vol.-\% $\mathrm{CO}_{2}$ at different temperatures 


\section{Specification of the $\mathrm{CO} 2$ gas sensor}

\begin{tabular}{|c|c|}
\hline Parameter & Value \\
\hline \multirow[t]{2}{*}{ concept } & $\begin{array}{l}\text { optical double beam } \\
\text { configuration }\end{array}$ \\
\hline & $\begin{array}{l}\text { atmosphere pressure } \\
\text { compensated }\end{array}$ \\
\hline measuring gas & $\mathrm{CO}_{2}$ \\
\hline $\begin{array}{l}\text { measurement } \\
\text { range }\end{array}$ & $0 \ldots 20$ Vol- $\%$ \\
\hline $\begin{array}{l}\text { measuring } \\
\text { temperature range }\end{array}$ & $0 \ldots 190^{\circ} \mathrm{C}$ \\
\hline $\begin{array}{l}\text { measurement } \\
\text { accuracy }\end{array}$ & $\begin{array}{l} \pm 0.2 \text { Vol- } \%+2 \% \text { of } \\
\text { read }\end{array}$ \\
\hline additional errors & $\begin{array}{l}<2 \% \text { of the } \\
\text { measured value for } \\
\text { temperature and } \\
\text { atmospheric pressure }\end{array}$ \\
\hline response time & $<20 s$ \\
\hline heat up time & $<5 \min$ \\
\hline $\begin{array}{l}\text { temperature range } \\
\text { sensor }\end{array}$ & $-25 \ldots+190^{\circ} \mathrm{C}$ \\
\hline $\begin{array}{l}\text { temperature range } \\
\text { ext. electronics }\end{array}$ & $-25 \ldots+80^{\circ} \mathrm{C}$ \\
\hline Interfaces & $\begin{array}{l}4-20 \mathrm{~mA} \\
0-1 \mathrm{~V}, 0-10 \mathrm{~V} \\
\mathrm{RS} 232, \mathrm{CAN}\end{array}$ \\
\hline Supply Voltage & $12 \mathrm{~V} \pm 1 \mathrm{~V} \max .2 \mathrm{~W}$ \\
\hline
\end{tabular}

\section{Conclusion}

Gas measuring modules normally work at room temperature conditions. This demonstration model is the basis for customized gas measurement cells and shows Micro-Hybrid's competence in high temperature and hermetic packaging technologies as well in the gas sensor design and calibration. The performance of the system is a feature based on MHE own infrared components. The thermopile detector consists of highly effective $\mathrm{BiSb} / \mathrm{Sb}$ thermocouples which enables a high sensitivity also at high temperatures. The emitter based on thermally stable nano-amorphous diamond-like carbon coatings. This structure enables high membrane temperatures up to $850{ }^{\circ} \mathrm{C}$ as well as high radiation intensities. The described gas sensor module is cost effective and best suited for harsh environments field applications like high temperature, high humidity and solvent atmospheres. It will undoubtedly find its place in the market of gas measurement modules.

\section{References}

[1] Biermann, S., Magi, A., Sachse, P.: Europäische Patentanmeldung, Hermetisches gasdichtes optoelektrisches oder elektrooptisches Bauteil sowie Verfahren zu seiner Herstellung, EP 2803961.

Europäisches Patentamt 19.11.2014

[2] Binder $\mathrm{GmbH}$.: Produktkatalog $\mathrm{CO} 2$ Inkubatoren Serie CB - NEU 60. 28.01.2015

[3] Budzier, H., Gerlach, G.: Thermische Infrarotsensoren: Grundlagen für Anwender. WILEY-VCH Verlag Weinheim 2010

[4] Gittler, E., Biermann, S., Brode, W., Störzner, F.: PCT Anmeldung, Verfahren zur Herstellung von in ein Gehäuse hermetisch dicht einlötbaren Fensterelementen und danach hergestellte Freiformfensterelemente, WO 2014/183746 A1. WIPO 20.11.2014

[5] Hoffmann, M., Müller, L., Käpplinger, I., Biermann, S., Brode, W.: Highly efficient IRemitters based on oriented nanostructures on silicon templates. Proceedings of 40th Micro and Nano Engineering (MNE 2014), Lausanne Switzerland, 22. - 26.09.2014

[6] Jäckle, J.: Über die Ursache der Thermopannung. http://www.uniuonstanz.de/FuF/Physik/Jaeckle/papers/therm ospannung/index.html 1998

[7] Morelli, D.M.: Interference Filter Handbook. 2. Aufl. JDS Uniphase Corporation (2006)

[8] Staab, J.: Industrielle Gasanalyse. Oldenburg Verlag 1994

[9] Stöcker, H.: Taschenbuch der Physik 5. Auflage. Harri Deutsch Verlag Frankfurt am Main 2004

[10] Spectrogon AB: Filter CWL shift as function of $\mathrm{AOI}$ and temperature. http://www.spectrogon.com/productservices/optical-filters/filter-cwl-shift-asfunction-of-aoi-and-temperature (2015) 